\title{
The Effect of Allopurinol on Renal Outcomes in Patients with Diabetic Kidney Disease: A Systematic Review and Meta-Analysis
}

\author{
Binbin Wu Lili Chen Yuankai Xu Qingqing Duan Zhibo Zheng \\ Zhigui Zheng Dongyuan He
}

Department of Nephrology, Zhejiang Hospital, Hangzhou, China

\section{Keywords}

Allopurinol - Diabetic kidney disease - Estimated glomerular filtration rate Albuminuria $\cdot$ Meta-analysis

\begin{abstract}
Background: Hyperuricemia is an independent risk factor for diabetic kidney disease (DKD) progression. Previous animal and cohort studies have reported that allopurinol administration could be of therapeutic benefit in diabetic subjects. However, there has been controversy regarding the effects of allopurinol on DKD. Objectives: The aim of our study was to investigate the efficacy of allopurinol on renal function in patients with DKD by meta-analysis of randomized controlled trials. Method: PubMed, EMBASE, and the Cochrane Library were searched from inception to October 2020. The primary outcome was a change in glomerular filtration rate (GFR). The secondary outcome was the change in albuminuria and serum uric acid (UA). Two reviewers independently assessed for risk of bias and extracted data. Standardized mean difference (SMD) or weighted mean difference (WMD) was calculated with random effects models and was reported with corresponding 95\% confidence intervals (Cls). Grading of Recommendations Assessment, Development, and Evaluation (GRADE) of the evidence was performed after meta-analysis. International prospective regis-
\end{abstract}

ter of systematic reviews registration CRD42020219132. $\boldsymbol{R e}$ sults: From 642 potentially relevant citations, 3 studies were ultimately included. Our results showed evident reduction in serum UA after allopurinol intervention (WMD $=-103.80$, $\left.95 \% \mathrm{Cl}-159.05,-48.55, R^{2}=76 \% ; p=0.04\right)$, with a high GRADE of evidence. However, allopurinol did not significantly improve GFR (WMD = 1.07, 95\% Cl -1.68, 3.82, $\ell^{2}=33 \% ; p$ $=0.45$ ), with a moderate GRADE of evidence. There was no significant difference on improvement of albuminuria in patients of allopurinol and those in placebo groups (SMD = $-0.26,95 \% \mathrm{Cl}-1.03,0.52, I^{2}=94 \% ; p=0.52$ ), with a moderate GRADE of evidence. Conclusions: The present research showed that allopurinol did not significantly improve renal function and albuminuria in patients with DKD.

\footnotetext{
(C) 2022 The Author(s).

Published by S. Karger AG, Basel
}

\section{Introduction}

The worldwide prevalence of diabetes mellitus (DM) has rapidly increased in the past 30 years and diabetic kidney disease (DKD) is a common microvascular diabetes complication. About $50 \%$ of patients with DKD progress to end-stage renal failure (ESRD) [1]. In light of the widespread prevalence and enormous financial costs, clinical
Karger@karger.com www.karger.com/kbr

Karger $\stackrel{\text { ' }}{5}$

GOPEN ACCESS
(C) 2022 The Author(s)

Published by S. Karger AG, Basel

This is an Open Access article licensed under the Creative Commons Attribution-NonCommercial-4.0 International License (CC BY-NC) (http://www.karger.com/Services/OpenAccessLicense), applicable to the online version of the article only. Usage and distribution for commercial purposes requires written permission.
Correspondence to:

Dongyuan He, hdy331@163.com 
management of DKD is of great societal relevance. Blood pressure control, glycemic and dyslipidemia control, weight reduction, and diet are contemporary managements recommended by The Kidney Disease Improving Global Outcomes clinical practice guidelines [2]. Although the above advances have meaningfully improved outcomes for cardiovascular disease, these improvements have not translated nearly as well to DKD $[3,4]$. Promising new therapies are of urgent need worldwide.

Over the last decade, researchers have found a significant association between higher levels of serum uric acid (UA) and worsening renal function. Results of cohort studies demonstrated that initial hyperuricemia is an independent risk factor for DKD progression [5, 6]. Ficociello et al. [7] found a relation between high-normal serum UA and risk of progressive renal function loss in patients with type $1 \mathrm{DM}$ (T1DM). UA has several reported effects by which it may cause $\mathrm{DKD}$, including endothelial dysfunction, increased activity of the renin-angiotensin-aldosterone system (RAAS), and induction of inflammatory cascades, in addition to profibrotic cytokine activation all of which have been demonstrated to contribute to the progression of microvascular disease and, thereby, renal injury in DKD [8].

Accumulating epidemiological evidence suggests that xanthine oxidase inhibitors, such as allopurinol, could be of therapeutic benefit in diabetic patients [9], but the role of allopurinol for the renal function is still controversial. Several researchers revealed that a reduction of UA with allopurinol might decrease the rate of glomerular filtration rate (GFR) loss. Liu et al. [10] reported that the allopurinol therapy was more effective in reducing serum UA and increasing GFR compared to the conventional treatment. Golmohammadi et al. [11] found GFR increased significantly after 12 months of allopurinol administration in patients with chronic kidney disease (CKD). However, results of randomized, placebo-controlled, doubleblinded, crossover trial implicated no significant association between allopurinol treatment and increasing GFR $[12,13]$. Therefore, we conducted a systematic review and meta-analysis of randomized controlled trials (RCT) to explore the effect of allopurinol on renal outcomes in patients with DKD.

\section{Methods}

\section{Protocol}

The prespecified protocol of our study was registered in the international prospective register of systematic reviews (PROSPERO), CRD42020219132 trial. The reports were based on the Pre- ferred Reporting Items for Systematic Reviews and Meta-Analyses Statement recommendations [14].

\section{Search Strategy and Inclusion Criteria}

We searched the PubMed, Embase, and the Cochrane Library from inception to October 2020, without language restrictions to identify RCTs that reported the efficacy of allopurinol compared with placebo in patients with DKD. The keywords of the first step were "diabetes mellitus" OR "diabetic nephropathy" OR "diabetic kidney disease" AND "allopurinol." The full literature search strategy was provided in the online supplementary Table (for all online suppl. material, see www.karger.com/doi/10.1159/000522248). Studies were included if they met the following criteria: (1) RCT design, (2) patients 18 years of age or older with DKD (urine albumin-to-creatinine ratio $\geq 30 \mathrm{mg} / \mathrm{g}$, or urine albumin-to-creatinine ratio $\geq 30 \mathrm{mg} / 24 \mathrm{~h}$, or urinary albumin excretion rate [UAER] $>0.2$ $\mathrm{mg} / \mathrm{min}$ or GFR $<60 \mathrm{~mL} / \mathrm{min} / 1.73 \mathrm{~m}^{2}$ ), (3) comparing the allopurinol with placebo, and (4) the prespecified outcomes of interest were reported separately for intervention and placebo groups. The exclusion criteria were as follows: (1) animal studies, nonrandomized design, cohort studies, case reports, (2) patients with the diagnosis of non-DKD, such as IgA nephropathy, membranous nephropathy, focal segmental glomerular sclerosis, minimal change disease, or lupus nephritis, and (3) studies with patients who had renal transplantation or estimated GFR $<15 \mathrm{~mL} / \mathrm{min} / 1.73 \mathrm{~m}^{2}$.

\section{Data Extraction}

Lili Chen and Yuankai Xu independently screened the search results and extracted data from each included study: trial characteristics (first author, publication year, country, sample size, and follow-up period), participants' baseline (age, male ratio, type of $\mathrm{DM}$, and duration of DM). The primary outcome was a change in estimated GFR from baseline till the end of the allopurinol intervention. The secondary outcome was the change in albuminuria and serum UA. The accuracy of the data was further confirmed by Qingqing Duan.

\section{Risk of Bias Assessment}

Zhibo Zheng and Zhigui Zheng independently assessed the methodological quality of the included studies. The risk of bias was estimated using the Cochrane Collaboration's "risk of bias" assessment tool [15]. We assessed 7 aspects: (1) random sequence generation, (2) allocation concealment, (3) blinding of participants and personnel, (4) blinding of outcome assessment, (5) incomplete outcome data, (6) selective reporting, and (7) other bias.

\section{Statistical Analysis}

We performed the data analysis using the software package Review Manager Version 5.4.0 software (Cochrane Collaboration). The standard deviation was imputed by interquartile ranges or full ranges when not available directly. The standardized mean difference was used to pool results from studies that reported untransformed changes in UAER and serum UA. Heterogeneity was assessed by the $I^{2}$ measure. We performed a meta-analysis using random effect models. The level of evidence was applied to the Grading of Recommendations Assessment, Development and Evaluation (GRADE) criteria and reported. $p<0.05$ was considered statistically significant. 
Fig. 1. Flow diagram for study selection of allopurinol versus control included in the meta-analysis.

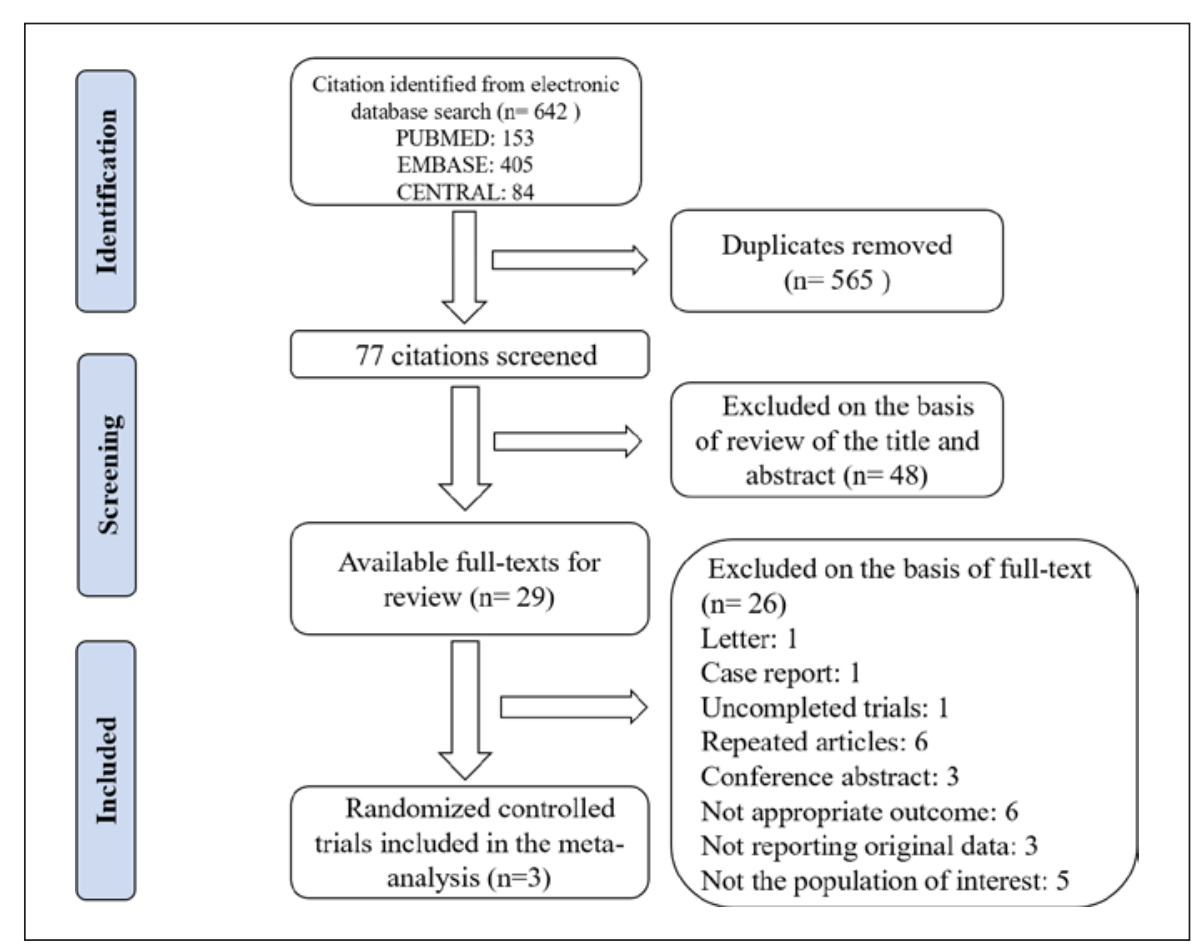

\section{Results}

\section{Search Results}

Figure 1 illustrates the flowchart for study inclusion and exclusion. We initially acquired 642 records through an online database search and deleted $565 \mathrm{du}$ plicates. Of these, 48 studies were excluded after evaluation of title and abstracts. We selected 29 full-text articles for detailed assessment for eligibility. Among these, we excluded 26 studies: 6 studies were lack of complete information ( 1 case report, 1 letter, 1 uncompleted trial, 3 conference abstracts), 6 studies due to repeated reports, 6 studies owing to lack of appropriate outcome (renal outcomes), 3 studies not reporting original data, 5 studies due to a lack of relevant population. Finally, we included 3 RCTs reporting the effects of allopurinol in diabetic patients.

\section{Characteristics of the Included Studies}

Three double-blind, placebo-controlled randomized clinical trials have been published as full papers, and the characteristics of the included studies are reported in Table 1 . The studies evaluated the effects of allopurinol in 722 diabetic patients (mostly, middle-aged men and women). Two studies included patients with type $2 \mathrm{DM}$ (T2DM), whereas only one study included patients with
T1DM. Patients' duration of DM ranged from 5 to 35 years and the follow-up period ranged from 21 to 38 months.

\section{Timepoint}

We choose the time point at the end of the intervention period used for the renal function, albuminuria, and other outcome measurements.

\section{Effects of Allopurinol on Kidney Function}

Two studies $(n=682)$ reported on effects of allopurinol on kidney function. There were no significant changes observed in estimated GFR with the effects of allopurinol $(1.07,95 \%$ confidence intervals (CI) $-1.68,3.82 ; p=$ 0.45, shown in Fig. 2). We found no significantly evident heterogeneity across the included studies $\left(I^{2}=33 \%, p=\right.$ 0.22 ). The level of evidence by GRADE was moderate, as an inconsistency was observed.

\section{Effects of Allopurinol on Albuminuria}

Two different measurements of protein excretion were described: UAER $(\mu \mathrm{g} / \mathrm{min})$ and albuminuria $(\mathrm{mg} / 24 \mathrm{~h})$. Therefore, the standardized mean difference was used to compare these diverse measures. Pooled analysis from all 3 studies $(n=722)$ showed no significant change in albuminuria after allopurinol treatment $(-0.26,95 \% \mathrm{CI}-1.03$ 


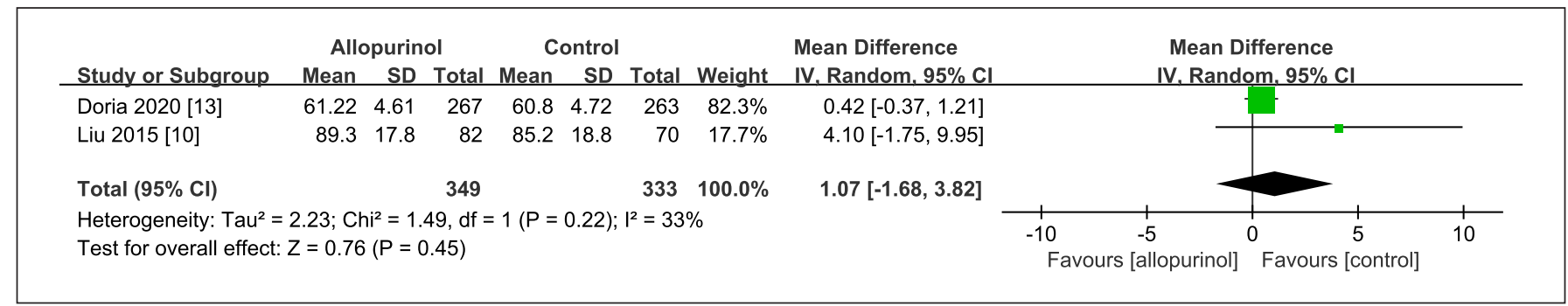

Fig. 2. The forest plot of estimated GFR for allopurinol intervention versus control.

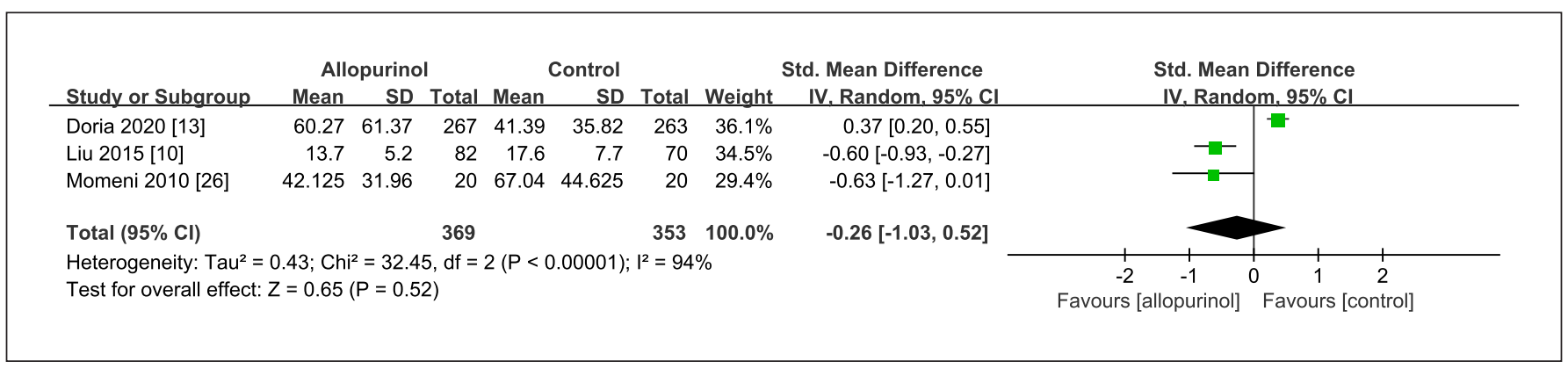

Fig. 3. The forest plot of albuminuria for allopurinol intervention versus control.

Table 1. Baseline characteristics of studies included in the meta-analysis

\begin{tabular}{|c|c|c|c|c|c|c|c|c|}
\hline Doria et al. [13] & $\begin{array}{l}\text { USA, Canada, } \\
\text { and Denmark }\end{array}$ & 530 & 38 & $34.6 \pm 12.3$ & & & & T1DM \\
\hline Liu et al. [10] & China & 152 & 36 & $5.0 \pm 1.8$ & & & & T2DM \\
\hline Momeni et al. [26] & Isfahan & 40 & 21 & $12.6 \pm 6.7$ & & & & T2DM \\
\hline Study & Mean age, years & Male, $\%$ & allopurinol & control & allopurinol & control & allopurinol & control \\
\hline Doria et al. [13] & $51.1 \pm 10.9$ & $351(66.2)$ & $41.1(7.7-216.0)$ & $43.0(9.0-198.0)$ & $6.1 \pm 1.5$ & $6.1 \pm 1.5$ & $75.4 \pm 18.7$ & $74.0 \pm 19.4$ \\
\hline Liu et al. [10] & $50.5 \pm 10.7$ & $70(46)$ & $12.3 \pm 3.7$ & $12.0 \pm 3.8$ & $7.3 \pm 0.2$ & $7.3 \pm 0.2$ & $90.1 \pm 17.5$ & $90.1 \pm 18.4$ \\
\hline Momeni et al. [26] & $57.7 \pm 10.5$ & $18(45)$ & - & - & $5.9 \pm 1.2$ & $6.5 \pm 2.2$ & - & - \\
\hline
\end{tabular}

DM, diabetes mellitus; T1DM, type 1 diabetes mellitus; T2DM, type 2 diabetes mellitus; UAER, urine albumin excretion rate; eGFR, estimated glomerular filtration rate.

to $0.52 ; p=0.52$; shown in Fig. 3). There is a significant heterogeneity $\left(I^{2}=94 \%, p<0.00001\right)$. The evidence for this finding by GRADE was moderate due to inconsistency.

\section{Effects of Allopurinol on Serum UA}

The results of the PERL study showed that serum UA level, which remained at baseline levels in the placebo group, decreased progressively in the allopurinol group from $6.1 \mathrm{mg}$ per deciliter at baseline to $3.7 \mathrm{mg}$ per deciliter at 16 weeks and remained at that level for the duration 


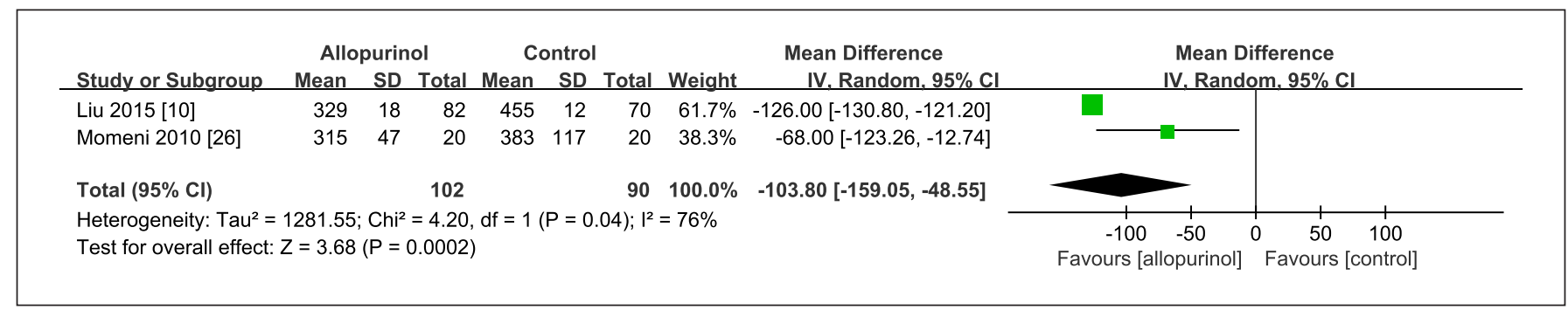

Fig. 4. The forest plot of changes in serum UA for allopurinol intervention versus control.

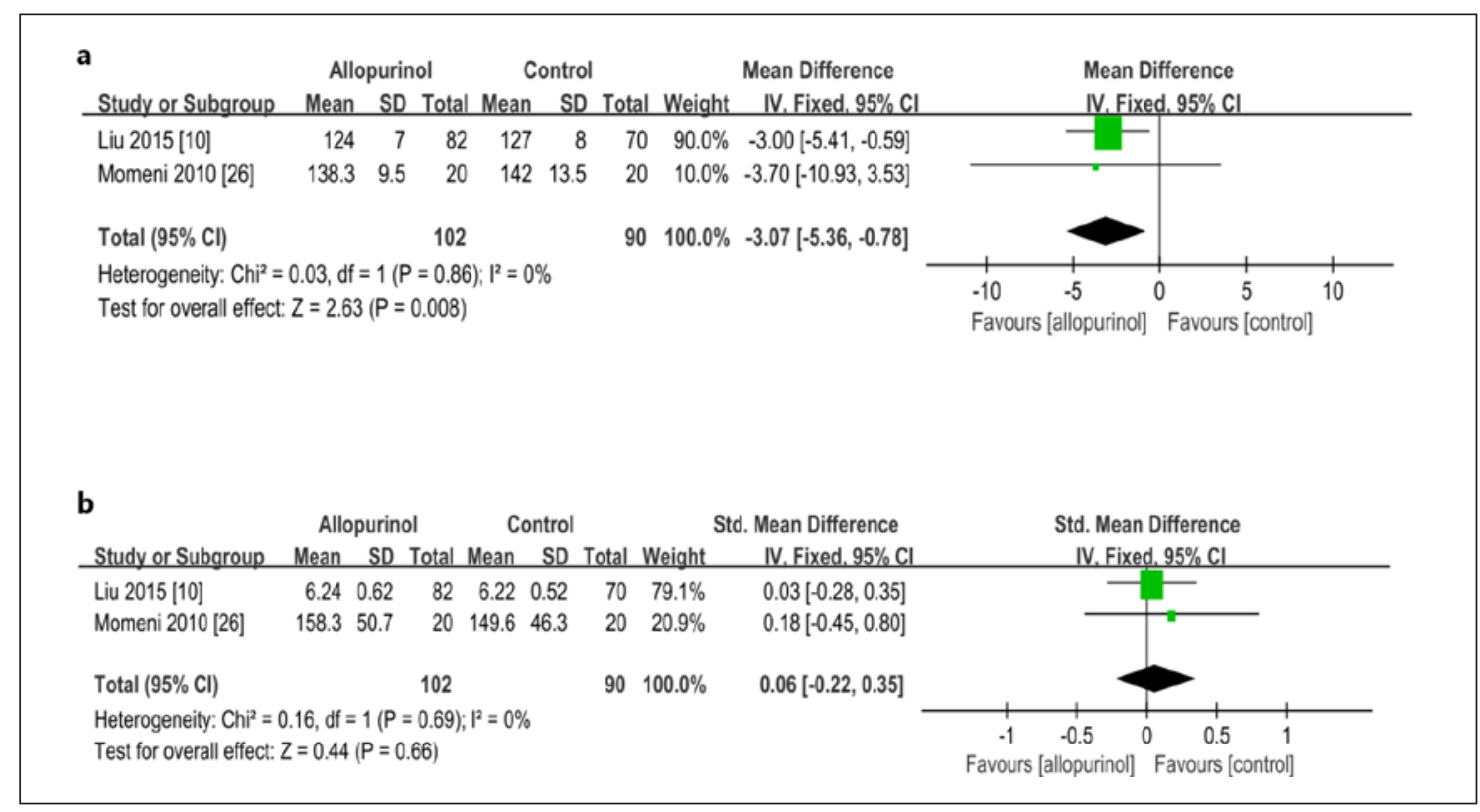

Fig. 5. The forest plot of blood pressure for allopurinol versus control: systolic blood pressure (a), diastolic blood pressure (b).

of the intervention period. However, the PERL study was excluded from the analysis due to a lack of sufficient original information.

We converted $\mathrm{mg} / \mathrm{dL}$ to $\mu \mathrm{mol} / \mathrm{L}$ so that the weighted mean difference-based approach can be used to derive a pooled treatment effect. Two studies $(n=192)$ provided details of allopurinol intervention on serum UA. According to the Figure 4, the weighted mean difference demonstrated evident reduction in serum UA after allopurinol intervention $(-103.80,95 \%$ CI -159.05 to -48.55 ; $p=$ $0.04)$ and the heterogeneity was also significant $\left(I^{2}=76 \%\right.$, $p<0.0002)$. The evidence for this finding by GRADE was high and unlikely to change in the future.

\section{Effects of Allopurinol on Blood Pressure}

Based on the included 2 study arms $(n=192)$ that reported absolute net or percentage changes in blood pressure, the meta-analysis revealed that allopurinol treatment was associated with a significant decrease in systolic blood pressure and diastolic blood pressure of 3.07 $\mathrm{mm} \mathrm{Hg}\left(95 \% \mathrm{CI}-5.36\right.$ to $\left.-0.78 ; p=0.008, I^{2}=0 \%\right)$ and $2.58 \mathrm{~mm} \mathrm{Hg}$ (95\% CI -4.40 to $-0.75 ; p=0.006, I^{2}=26 \%$ ), respectively (Fig. 5). The level of evidence by GRADE was moderate due to the uncertainty.

\section{Effects of Allopurinol on Additional Outcomes}

As for additional outcomes described in the posted protocol, such as HbA1c, ESRD, cardiovascular events, and all-cause mortality, we could not draw any conclu- 


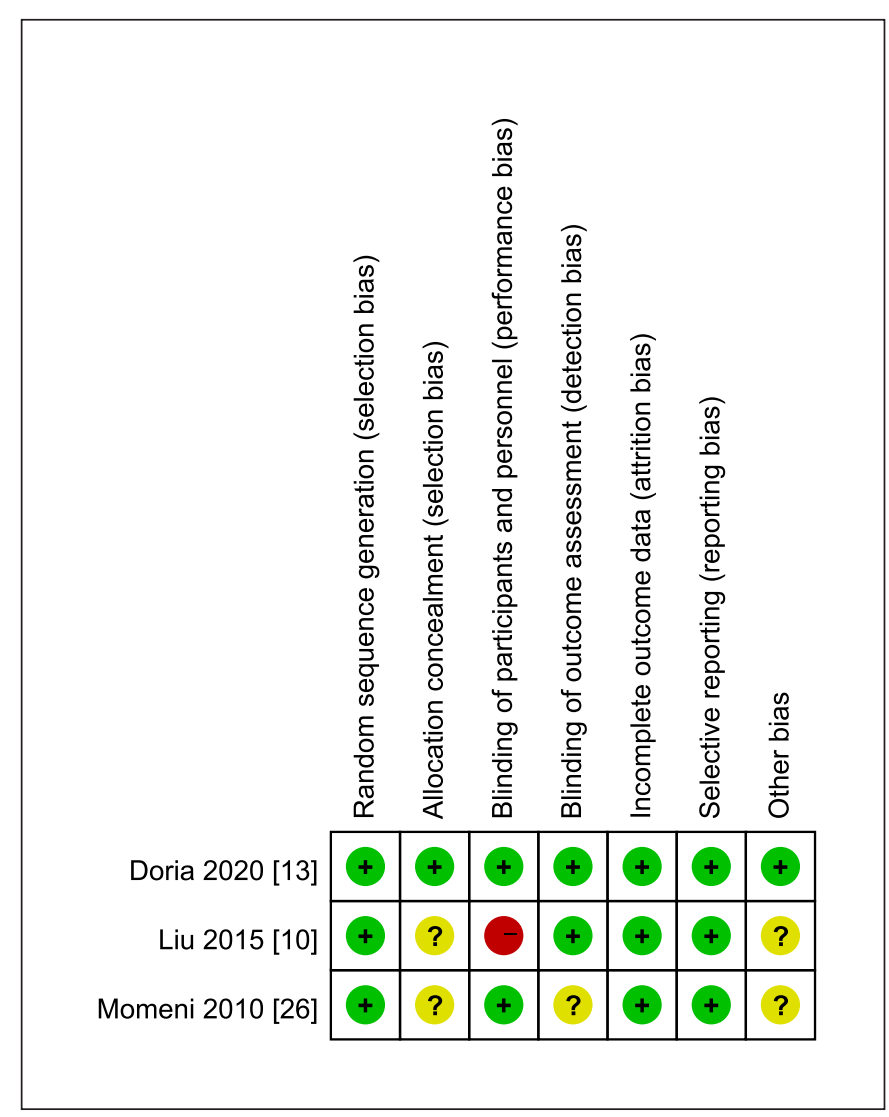

Fig. 6. Risk of bias summary: review authors' judgments about each risk of bias item for each included RCT study. RCT, randomized controlled trials. sion due to a lack of original information. Neither Liu et al. [10] nor Doria et al. [13] reported treatment effects of allopurinol on HbAlc. Only Doria et al. [13] reported treatment effects of allopurinol on end-stage kidney disease and cardiovascular events. None of them reported all-cause mortality.

\section{Risk of Bias}

The outcomes of the Cochrane Collaboration's tool for assessing the risk of bias are presented in Figures 6 and 7 . As expected, only 1 full-paper trial was of high quality. Potential bias may come from the lack of blinding in 1 study, as well as from the unclear description of allocation concealment and other bias.

\section{Discussion}

In this meta-analysis, we included 3 RCTs that investigated the efficacy of allopurinol intervention in patients with type 1 or $2 \mathrm{DM}$. The results of our research indicated that, when compared with placebo, treatment with allopurinol demonstrated no renal protective effect on DKD neither on improving GFR $\left(1.07,95 \% \mathrm{CI}-1.68,3.82, I^{2}=\right.$ $33 \%$; $p=0.45)$ nor albuminuria $(-0.26,95 \% \mathrm{CI}-1.03$, $0.52, I^{2}=94 \% ; p=0.52$ ).

For most of the combined data, there is relatively limited precision in the pooled estimates. The GRADE recommends examining the $95 \%$ CI first. Quality of evidence would be downgraded when the clinical practice was in-

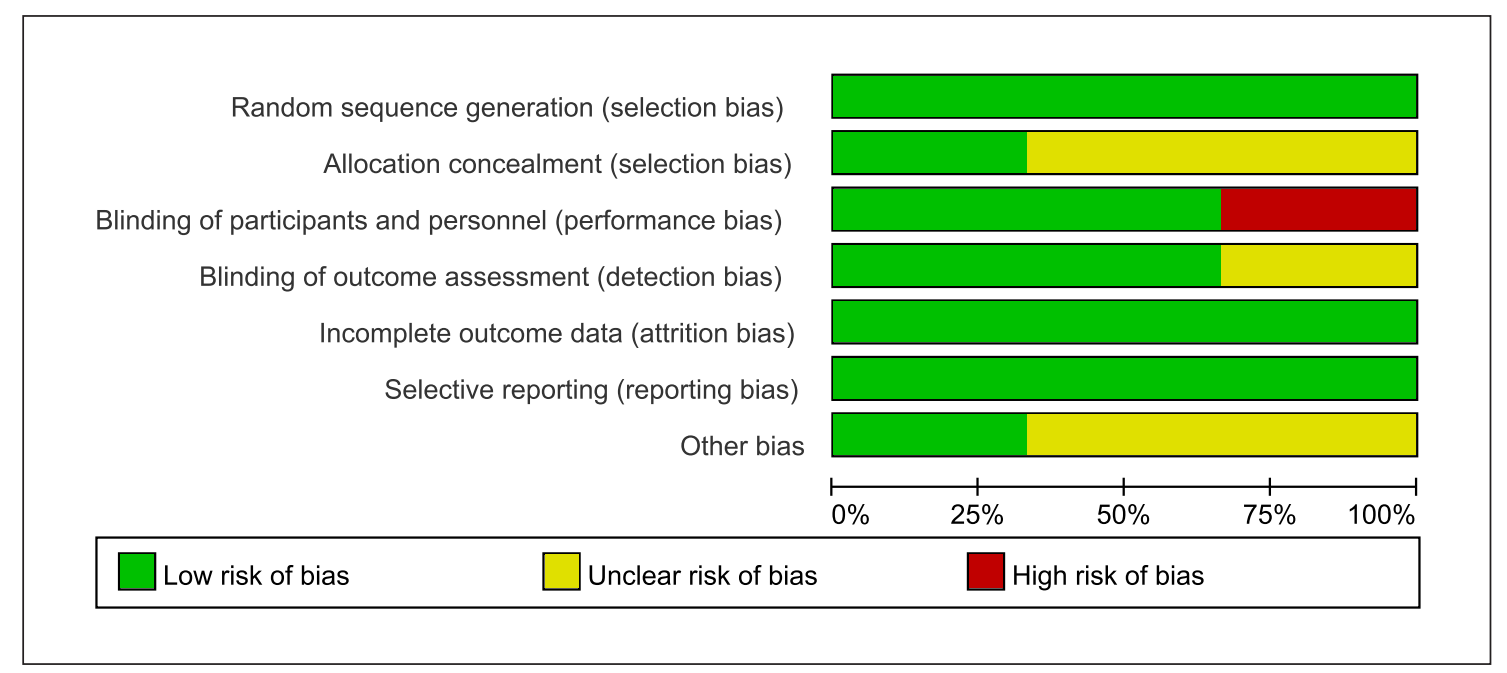

Fig. 7. Risk of bias graph: review authors' judgments about each risk of bias item presented as percentages across all included RCT studies. RCT, randomized controlled trials. 
compatible with the $95 \%$ CI. For continuous variables, it is important to calculate the optimal information sample. It will consider downgrading the quality of evidence for imprecision due to a low number of events accompanied by a large effect size.

It is necessary to explain the timepoints at which measurements were taken in the included studies. We choose the time point at the end of the intervention period used for the renal function, albuminuria, and other outcome measurements. There are 2 reasons for the choice. First, this timepoint of the PERL study (the largest contributing study) is consistent with another 2 studies, which makes the pooled analysis convincing. Then, the metabolite of allopurinol, oxypurinol, has a half-life of 12-17 h. Based on the half-life, it is likely that allopurinol was no longer effective following the washout period. Based on the above characteristics, the time point at the end of the intervention period seems more suitable.

$\mathrm{DKD}$ is the leading cause of progressive kidney disease and the increasing global prevalence has prompted great efforts to tackle the growing epidemic. Prevention of DKD is a major public health challenge worldwide. Despite some progress in reducing cardiovascular complications (through intensive blood pressure and glycemic control, lifestyle modifications, weight reduction and diet, and the use of rennin angiotensin system inhibitors), the rate of CKD progression to ESRD has remained unchanged over the past 2 decades in patients with DM [4, 16]. The identification of new effective treatment is of urgent medical needs. Allopurinol, a competitive xanthine oxidase inhibitor, was traditionally used for the management of gout in addition to reducing serum UA levels. It has been reported that allopurinol has an antioxidant property that might partially reverse endothelial dysfunction $[17,18]$. There has been renewed interest in the role of allopurinol in the management of cardiovascular disease in the last decade [19] and the potential to be a novel therapy for the treatment of DKD. However, a large prospective cohort study revealed that allopurinol was not associated with an improvement of renal function in patients with CKD [20]. The effect of allopurinol on DKD still remained inconsistent, and the results of our study demonstrated a great meaning.

An elevated level of UA can lead to arteriolopathy of preglomerular vessels, endothelial dysfunction, as well as glomerular hypertension in animal models [21]. Also, hyperuricemia is associated with persistent macroalbuminuria in patients with DM. Since hyperuricemia was recognized to be a risk factor for nephropathy [22], a growing number of studies have explored the role of allopurinol in the diabetic subject. Kosugi et al. [23] have evaluated the effects of allopurinol in diabetic mice. They found that hyperuricemia has a pathogenic role in mild tubulointerstitial injury and allopurinol treatment could reduce albuminuria and ameliorate tubulointerstitial injury. One possible mechanism is the reduction of UA might inhibit nucleotide bindings and oligomerization domain-like receptor family pyrin domain-containing 3 inflammasome activation, which could induce kidney inflammation and result in the generation and development of DKD [24]. Momeni et al. [25] conducted an RCT in patients with T2DM and concluded low-dose allopurinol could reduce the severity of proteinuria $(1,011 \pm 767$ $\mathrm{mg} / 24 \mathrm{~h}$ in allopurinol group vs. 1,609 $\pm 1,071 \mathrm{mg} / 24 \mathrm{~h}$ in the placebo group, $p=0.049$ ). The study published in clinical endocrinology indicated long-term allopurinol treatment was benefit to effective control of serum UA ([329 \pm 18 vs. $455 \pm 12] \mu \mathrm{mol} / \mathrm{L}, p<0.001$ ), contributing to a decrease in UAER ([13.7 \pm 5.2 vs. $17.6 \pm 7.7] \mu \mathrm{g} / \mathrm{mL}$, $p=0.002)$ when compared to the conventional treatment [10].

Unfortunately, a few clinical studies have reported disappointing results. A randomized, placebo-controlled, double-blinded study that included 30 individuals with T1DM found no clear benefits on renal parameters from allopurinol treatment [26].

They concluded that short-term UA lowering did not improve UAER or GFR and called for large multicenter RCT to investigate the effect of allopurinol treatment on albuminuria and GFR. Another randomized, doubleblinded, crossover trial also demonstrated no difference in UAER and GFR compared with placebo [12]. Elbarbary et al. [27] enrolled 90 type 1 diabetic adolescents to assess the short-term effect of allopurinol treatment and reported that low-dose allopurinol was not effective in reducing microalbuminuria. The result of Preventing Early Renal Loss in Diabetes Study was published in The New England Journal of Medicine in 2020. Doria et al. [13] found no evidence of clinically meaningful benefits of serum UA reduction with allopurinol on renal outcomes among patients with T1DM and early-to-moderate DKD.

Interventions of DKD mainly consist of glycemic control, blood pressure control, and RAAS inhibition. Whether intensive glycemic control protects kidney health in the long term has been no uniform conclusion. The National Kidney Foundation's Kidney Disease Outcomes Quality Initiative (KDOQI) guideline generally suggests keeping $\mathrm{HbA} 1 \mathrm{c}$ levels to $7.0 \%$ or less for patients with type 1 or T2DM. EDIC (Epidemiology of Diabetes Interventions and Complications) study indicated that 
intensive DM therapy reduced the risk of an impaired GFR by $50 \%$ [28]. The Action in Diabetes and Vascular Disease: Preterax and Diamicron MR Controlled Evaluation Trial [29] reported intensive glycemic control was associated with improvements in kidney outcomes (a $65 \%$ reduction in ESRD). Contrasting findings were reported in the ACCORD Study, with a 7\% higher rate of doubling of serum creatinine, or a $>20 \mathrm{~mL} / \mathrm{min}$ per 1.73 $\mathrm{m}^{2}$ decrease in estimated GFR and no effect found on the development of ESRD [30].

The roles of blood pressure in managing $\mathrm{DKD}$ remain controversial despite decades of study. There are no rigorous clinical trial data to support strict blood pressure control for kidney protection in patients with T2DM. The Action to Control Cardiovascular Risk in Diabetes (ACCORD) Study [30] reported that aggressive blood pressure control (mean achieved, 119 vs. $134 \mathrm{~mm} \mathrm{Hg}$ ) could reduce the risk of developing microalbuminuria but not kidney failure (defined as a serum creatinine $>3.3 \mathrm{mg} / \mathrm{dL}$, dialysis, or kidney transplantation). In the landmark UK Prospective Diabetes Study Group (UKPDS) trial of patients with T2DM and newly diagnosed hypertension, randomization to intensive versus less tight blood pressure control (mean levels achieved, $144 / 82$ vs. $154 / 87 \mathrm{~mm}$ $\mathrm{Hg}$ ) did not significantly reduce the risk of developing kidney failure [31].

The results of high-quality RCTs supported the use of RAAS blockers in patients with DKD could delay GFR decline. The IDNT Study [32] reported the rate of decline in the estimated GFR was 5.5, 6.8, and $6.5 \mathrm{~mL} / \mathrm{min}$ per $1.73 \mathrm{~m}^{2}$ per year in the irbesartan, amlodipine, and placebo arms, respectively. The results of The Reduction of Endpoints in NIDDM [33] with the Angiotensin II Antagonist Losartan Study indicated that Losartan therapy resulted in a reduced risk of ESRD or doubling of serum creatinine dropping by $25 \%$ and $28 \%$, respectively. Losartan reduced the median rate of decline in estimated GFR by $0.8 \mathrm{~mL} / \mathrm{min}$ per $1.73 \mathrm{~m}^{2}$ per year.

Although this is the first meta-analysis on this topic of the effectiveness of allopurinol in the management of diabetic patients and the present study was based on RCTs, it has several limitations. First, there was variation in the study subjects (type 1 or type $2 \mathrm{DM}$ ), different doses of allopurinol administration, outcome analysis (GFR, UA, and albuminuria), and duration of the study. These differences might explain some of the heterogeneity among the trials. Second, the total number of RCTs included is low. We found 4 double-blind, randomized, placebocontrolled studies in diabetic patients, published only in abstract or letter form. Further analyses (subgroup and sensitivity analysis) are not likely to be achieved due to the low number. Another limit is that this study assessed only the effectiveness of allopurinol on renal outcomes. The role of allopurinol played in cardiovascular disease, endothelial dysfunction, and inflammation was not an endpoint of this study, because previous literature has reported $[17,34]$.

\section{Conclusion}

In conclusion, our research indicates that allopurinol was not related to a significant improvement of renal function in patients with DKD. No significant UAER reduction was found after allopurinol treatment. In the future, large-scale and multicenter RCTs should be carried out to help clinicians better understand the actual effect of allopurinol on kidney outcomes in DM.

\section{Statement of Ethics}

An ethics statement is not applicable because this study is based exclusively on published literature.

\section{Conflict of Interest Statement}

The authors have no conflicts of interest to declare.

\section{Funding Sources}

No funding or grant support was obtained for this study.

\section{Author Contributions}

Lili Chen and Yuankai Xu collect relevant information. Binbin $\mathrm{Wu}$ and Dongyuan analyzed the data. Qingqing Duan, Zhibo Zheng, Zhigui Zheng have been involved in revising it critically for important intellectual content; Binbin Wu and Dongyuan wrote the paper. All authors read and approved the final manuscript.

\section{Data Availability Statement}

The data that support the findings of this study are available from the corresponding author. 


\section{References}

1 Kato M, Natarajan R. Epigenetics and epigenomics in diabetic kidney disease and metabolic memory. Nat Rev Nephrol. 2019;15(6): 327-45.

2 Doshi SM, Friedman AN. Diagnosis and management of type 2 diabetic kidney disease. Clin J Am Soc Nephrol. 2017;12(8): 1366-73.

3 Alicic RZ, Rooney MT, Tuttle KR. Diabetic kidney disease: challenges, progress, and possibilities. Clin J Am Soc Nephrol. 2017;12(12): 2032-45.

4 Fu H, Liu S, Bastacky SI, Wang X, Tian XJ, Zhou D. Diabetic kidney diseases revisited: a new perspective for a new era. Mol Metab. 2019;30:250-63.

5 Bartáková V, Kuricová K, Pácal L, Nová Z, Dvořáková V, Švrčková M, et al. Hyperuricemia contributes to the faster progression of diabetic kidney disease in type 2 diabetes mellitus. J Diabetes Complications. 2016;30(7): 1300-7.

6 Ito H, Abe M, Mifune M, Oshikiri K, Antoku $\mathrm{S}$, Takeuchi Y, et al. Hyperuricemia is independently associated with coronary heart disease and renal dysfunction in patients with type 2 diabetes mellitus. PLoS One. 2011; 6(11):e27817.

7 Ficociello LH, Rosolowsky ET, Niewczas MA, Maselli NJ, Weinberg JM, Aschengrau A, et al. High-normal serum uric acid increases risk of early progressive renal function loss in type 1 diabetes: results of a 6-year follow-up. Diabetes Care. 2010;33(6):1337-43.

8 Jalal DI, Maahs DM, Hovind P, Nakagawa T. Uric acid as a mediator of diabetic nephropathy. Semin Nephrol. 2011;31(5):459-65.

9 Pacher P, Nivorozhkin A, Szabó C. Therapeutic effects of xanthine oxidase inhibitors: renaissance half a century after the discovery of allopurinol. Pharmacol Rev. 2006;58(1):87114.

10 Liu P, Chen Y, Wang B, Zhang F, Wang D, Wang Y. Allopurinol treatment improves renal function in patients with type 2 diabetes and asymptomatic hyperuricemia: 3-year randomized parallel-controlled study. Clin Endocrinol. 2015;83(4):475-82.

11 Golmohammadi S, Almasi A, Manouchehri M, Omrani HR, Zandkarimi MR. Allopurinol against progression of chronic kidney disease. Iran J Kidney Dis. 2017;11(4):286-93.

12 Pilemann-Lyberg S, Persson F, Frystyk J, Rossing P. The effect of uric acid lowering treatment on albuminuria and renal function in type 1 diabetes: a randomized clinical trial. Diabet Med. 2018;35(3):392-3.
13 Doria A, Galecki AT, Spino C, Pop-Busui R, Cherney DZ, Lingvay I, et al. Serum urate lowering with allopurinol and kidney function in type 1 diabetes. N Engl J Med. 2020; 382(26):2493-503.

14 Liberati A, Altman DG, Tetzlaff J, Mulrow C, Gøtzsche PC, Ioannidis JP, et al. The PRISMA statement for reporting systematic reviews and meta-analyses of studies that evaluate health care interventions: explanation and elaboration. Ann Intern Med. 2009;151(4): W65-94.

15 Cumpston M, Li T, Page MJ, Chandler J, Welch VA, Higgins JP, et al. Updated guidance for trusted systematic reviews: a new edition of the Cochrane Handbook for Systematic Reviews of Interventions. Cochrane Database Syst Rev. 2019;10:ED000142.

16 Anders HJ, Huber TB, Isermann B, Schiffer M. CKD in diabetes: diabetic kidney disease versus nondiabetic kidney disease. Nat Rev Nephrol. 2018;14(6):361-77.

17 Alem MM. Allopurinol and endothelial function: a systematic review with meta-analysis of randomized controlled trials. Cardiovasc Ther. 2018;36(4):e12432.

18 Dogan A, Yarlioglues M, Kaya MG, Karadag Z, Dogan S, Ardic I, et al. Effect of long-term and high-dose allopurinol therapy on endothelial function in normotensive diabetic patients. Blood Press. 2011;20(3):182-7.

19 Okafor ON, Farrington K, Gorog DA. Allopurinol as a therapeutic option in cardiovascular disease. Pharmacol Ther. 2017;172:139_ 50.

20 Vargas-Santos AB, Peloquin CE, Zhang Y, Neogi T. Association of chronic kidney disease with allopurinol use in gout treatment. JAMA Intern Med. 2018;178(11):1526-33.

21 Hovind P, Rossing P, Johnson RJ, Parving HH. Serum uric acid as a new player in the development of diabetic nephropathy. J Ren Nutr. 2011;21(1):124-7.

22 Kushiyama A, Tanaka K, Hara S, Kawazu S. Linking uric acid metabolism to diabetic complications. World J Diabetes. 2014;5(6): 787-95.

23 Kosugi T, Nakayama T, Heinig M, Zhang L, Yuzawa Y, Sanchez-Lozada LG, et al. Effect of lowering uric acid on renal disease in the type 2 diabetic $\mathrm{db} / \mathrm{db}$ mice. Am J Physiol Renal Physiol. 2009;297(2):F481-8.

24 Qiu YY, Tang LQ. Roles of the NLRP3 inflammasome in the pathogenesis of diabetic nephropathy. Pharmacol Res. 2016;114:251-64.
25 Momeni A, Shahidi S, Seirafian S, Taheri S, Kheiri S. Effect of allopurinol in decreasing proteinuria in type 2 diabetic patients. Iran J Kidney Dis. 2010;4(2):128.

26 Pilemann-Lyberg S, Persson F, Frystyk J, Rossing P. The effect of uric acid lowering on albuminuria and renal function in type 1 diabetes: a randomized clinical trial. J Am Soc Nephrol. 2017;28:227.

27 Elbarbary N, El-Samahy M, Abo-El-Asrar M, Sallam D. Effect of allopurinol versus angiotensin converting enzyme inhibitors in decreasing microalbuminuria in type 1 diabetic patients. Horm Res Paediatr. 2016;86:211-2.

28 Epidemiology of Diabetes Interventions and Complications (EDIC). Epidemiology of Diabetes Interventions and Complications (EDIC). Design, implementation, and preliminary results of a long-term follow-up of the diabetes control and complications trial cohort. Diabetes care. 1999;22(1):99-111.

29 Perkovic V, Heerspink HL, Chalmers J, Woodward M, Jun M, Li Q, et al. Intensive glucose control improves kidney outcomes in patients with type 2 diabetes. Kidney Int. 2013;83(3):517-23.

30 Ismail-Beigi F, Craven TE, O’Connor PJ, Karl D, Calles-Escandon J, Hramiak I, et al. Combined intensive blood pressure and glycemic control does not produce an additive benefit on microvascular outcomes in type 2 diabetic patients. Kidney Int. 2012;81(6):586-94.

31 Intensive blood-glucose control with sulphonylureas or insulin compared with conventional treatment and risk of complications in patients with type 2 diabetes (UKPDS 33). UK Prospective Diabetes Study (UKPDS) Group. Lancet. 1998;352(9131):837-53.

32 Lewis EJ, Hunsicker LG, Clarke WR, Berl T, Pohl MA, Lewis JB, et al. Renoprotective effect of the angiotensin-receptor antagonist irbesartan in patients with nephropathy due to type 2 diabetes. N Engl J Med. 2001;345(12): 851-60.

33 Brenner BM, Cooper ME, de Zeeuw D, Keane WF, Mitch WE, Parving $\mathrm{HH}$, et al. Effects of losartan on renal and cardiovascular outcomes in patients with type 2 diabetes and nephropathy. N Engl J Med. 2001;345(12):8619.

34 Bredemeier M, Lopes LM, Eisenreich MA, Hickmann S, Bongiorno GK, d'Avila R, et al. Xanthine oxidase inhibitors for prevention of cardiovascular events: a systematic review and meta-analysis of randomized controlled trials. BMC Cardiovasc Disord. 2018;18(1): 24. 